\title{
El yoga: estrategia para la mejora de la convivencia y de la participación activa en la vida social de la institución educativa en la etapa de educación inicial
}

\author{
SILVIA MORATALLA ISASI ${ }^{*}$ \\ Junta de Comunidades de Castilla-La Mancha \\ ARÁNZAZU CARRASCO GALÁN** \\ Junta de Comunidades de Andalucía \\ CRISTINA SÁNCHEZ ROMERO ${ }^{* * *}$ \\ Universidad Nacional de Educación a Distancia (UNED) \\ Recibido el 26-10-2017; primera evaluación el 14 -12-2018; \\ segunda evaluación el 25-01-2019; aceptado el 07-02-2019
}

\section{Resumen}

Este artículo describe la experiencia realizada en un centro educativo con alumnos del $2^{\circ}$ ciclo de educación inicial $(\mathrm{N}=72)$ con los que se trabajó de forma fácil y divertida ejercicios de respiración y relajación propios de yoga. La aplicación en el aula casi a diario de estos ejercicios de forma práctica y lúdica permitió comprobar cómo adoptan los niños actitudes mucho más tranquilas, menos tensas y de más autocontrol. Además, favorece su potencial creativo y de talento. Es aconsejable que los alumnos se adentren en estos ejercicios a partir de los tres años en adelante,

\footnotetext{
* Doctora por la UNED. Inspectora de Educación de la Junta de Comunidades de Castilla-La Mancha Máster en Informática Educativa. Profesora de Pedagogía Terapéutica, profesora asociada en la UCLM. Cuerpos de Inspectores UCLM. Licenciada en Filosofía y Ciencias de la Educación, diplomada en Profesorado de EGB y profesora de Piano. Principales líneas de investigación: diversidad educativa, educación intercultural, formación de profesorado, TIC aplicadas a la educación especial. Correo: silvia.moratalla@gmail.com

** Profesora de Educación Infantil. Junta de Comunidades de Andalucía. Correo: aranzazuco1111@ hotmail.com

*** Autor de correspondencia. Doctora por la UNED. Máster en Informática Educativa, licenciada en Filosofía y Ciencias de la Educación. Cargo académico: secretaria de la Facultad de Educación. Profesora del Departamento de Didáctica, Organización Escolar y Didácticas Especiales (UNED) Secretaría Académica y profesora del Máster/Posgrado Oficial sobre Tratamiento Educativo de la Diversidad. Tutora Centro Asociado de Madrid con Venia Docendi. Correo: csanchez@edu.uned.es
} 
pues a esa edad los pequeños pueden, en su mayoría, comprender lo que se puede esperar y lo que se quiere de ellos. Además ya tienen la capacidad de realizar y controlar ciertos movimientos de su cuerpo.

Palabras clave: yoga, educación inicial, respiración y relajación.

\section{Yoga: strategy for the improvement of living together and active participation in social of the educational institution in the early childhood education stage}

\section{Abstract}

This article describes the experience in a school with pupils from four to five years of early childhood education $(\mathrm{N}=72)$ with whom I worked in an easy and funny way relaxation breathing exercises specific to yoga. The application in the classroom of these exercises almost every day in a practical and funny way allows children to see how attitudes take much calmer, less tense and more self-control. It also encourages their creative potential and talent. It is advisable for students getting into these exercises from four years onwards, because at that age, children know exactly what to expect and what you want from them. Besides, they have the ability to make and control certain body movements.

Keywords: Yoga, children's education, breathing and relaxation.

\section{Yoga: uma estratégia para melhorar a coexistência e participaçáo ativa na vida social da instituiçáo de ensino na etapa de educaçáo inicial}

\section{Resumo}

Este artigo descreve a experiência em um centro educacional com alunos do $2^{\circ}$ ciclo de educaçáo inicial $(\mathrm{N}=72)$ com os quais eles trabalharam com facilidade e divertidos exercícios de respiração e relaxamento de ioga. A aplicação na sala de aula quase diariamente desses exercícios de maneira prática e brincalhão nos permite ver como as crianças adotam as atitudes muito mais calmas, menos tensas e mais autocontrole. Também aumenta o seu potencial e talento criativo. É aconselhável que os alunos ingressem nesses exercícios a partir dos três anos de idade, porque naquela idade as crianças já sabem perfeitamente o que se pode esperar e o que é desejado. Além disso, eles já têm a capacidade de realizar e controlar certos movimentos de seu corpo

Palavras-chave: Yoga, educação inicial, respiração e relaxamento. 


\section{INTRODUCCIÓN}

La transición a la escuela está relacionada con el optimismo en los diferentes niveles educativos (Boman y Yates, 2001; Dawson y Pooley, 2013). Los estudiantes que llegan a un centro por primera vez presentan inquietud, inseguridad, estrés y falta de hábitos; esto influye en la práctica profesional y en el rendimiento escolar.

Las investigaciones de Chao, Hwu, y Chang (2011) describen que existe un creciente consenso de la investigación de niños y adolescentes sobre importantes factores protectores como son la vinculación y/o apego, las competencias, el optimismo, el contexto a través del concepto de resiliencia. Las experiencias vividas por los estudiantes y las críticas recibidas sobre todo por familiares, docentes y compañeros son factores que explicarían el desarrollo del optimismo en estos estudiantes.

La familia, principalmente, y los docentes, en segundo lugar, cobran una especial importancia sobre todo en sus primeros ańos de vida educativa. Estos son un soporte imprescindible para que el estudiante establezca bases seguras, a partir de las cuales pueda explorar, sentirse dueño de su propia conducta y aprender a ponerse límites (Gillham, Shatté, Reivich y Seligman, 2001; Gillham y Reivich, 2004; Hernangómez, 2002).

Ante esta situación, es importante que el docente sea optimista y trasmita ese optimismo a sus estudiantes, es decir, que sepa aprovechar los medios con los que cuenta, saber motivar y animar a los estudiantes y conseguir que aprendan, pues va a ser crucial para que estos puedan superar el stress y los desafíos que se les planteen cada día y encontrar un ambiente de clase más acogedor que les proporcione seguridad y tranquilidad. El ser optimista requiere reconocer lo mejor de una persona, de una situación, de una experiencia (Marujo, Neto y Perlorio, 2003).

En las investigaciones presentadas por Marujo, Neto, Caetano y Rivero (2007) y Marujo y Neto (2010), se analizan cómo se aborda el valor positivo en las organizaciones y en los lugares de trabajo, como un nuevo enfoque hacia las emociones positivas en los centros de trabajo.

Los docentes deben establecer estrategias sistemáticas para maximizar el aprendizaje que, a su vez, pueden ser desarrolladas por otros agentes educativos como la familia u otros especialistas del entorno del niño para el desarrollo de actividades y el establecimiento de rutinas dentro y fuera del entorno escolar (DEC, 2016). 
El docente, en la etapa de Educación Inicial, es importante que oferte y fomente experiencias de control y dominio. La sensación de control en el estudiante viene determinada por la confianza que tiene en sus propias capacidades y en la posibilidad de poder cambiar cosas en el entorno que le rodea. El estudiante en sus primeros ańos de vida recorre el entorno y lo manipula. Esa acción sobre el medio junto con el desarrollo del lenguaje y de nuevas capacidades cognitivas es algo que se puede favorecer durante la etapa de educación inicial, si los docentes y la familia le permiten explorar en entornos seguros en los cuales puedan asumir riesgos y resolver problemas. El optimismo en los estudiantes podría predecir un ajuste adecuado de los mismos en sus interacciones y actividades educativas (Boman y Yates, 2001; Ruthig, Haynes, Perry, y Chipperfield, 2007). En este sentido, el optimismo del docente ha sido estudiado por Hoy, Hoy y Kurz (2008) como optimismo académico en la eficacia y confianza en lo académico y optimismo disposicional en la gestión del aula, y en creencias y prácticas centradas en los estudiantes.

Entre los predictores exitosos en el resultado de aprendizaje de los nińos, recomendados por la National Association for the Education of Young Children en el período de la infancia, destaca la necesidad de hacer hincapié en desarrollar la autorregulación de los niños, su participación y su atención concentrada (NAEYC, 2009).

Los docentes deben enseñar a los estudiantes ante la incertidumbre y el conflicto, y asumir una práctica creativa para el desarrollo de la motivación, la esperanza y el optimismo en los estudiantes, porque los nińos, como afirman Janzen, Hildebrand, Kaufmann, y Saklofske (2001), pueden comunicar o mostrar sentimientos de infelicidad y tristeza, en algunos casos, pueden mostrar hasta depresión. Por ello, es importante desarrollar habilidades de autorregulación desde edades tempranas como nos indican Whitebread y Basilio (2012) para el desarrollo general y educativo de los niños. El optimismo como indica Seligman, Ernst, Gillham, Reivich, y Linkins (2009) es una herramienta que hace posibles logros individuales.

Como ya se ha dicho anteriormente, el ánimo, la motivación, el respeto y la moderación son muy importantes dentro de la práctica educativa diaria. Practicando el yoga como experiencia innovadora en el aula con los estudiantes, se va a contribuir al aumento de su bienestar, tranquilidad mental y liberación de tensiones de estrés. La práctica de yoga, como si fuera un juego de manera constante y gradual, les proporcionará una refrescante sensación de vitalidad y optimismo, es decir, les generará una actitud positiva y alegre para enfrentarse a las diferentes situaciones que se les presenten y les abrirá el camino del autoconocimiento. 


\section{MarCo teórico}

\subsection{El yoga en el aula de Educación Inicial}

Autores como Garfinkel y Schumacher (2000); Subramanyam (1989); Telles, Gaur, y Balkrishna (2009) describen el yoga como la disciplina y conjunto de técnicas que ayudan al bienestar de la persona. Respecto a los niños, concretamente, el estudio de White (2009) describe esta técnica como para calmar la mente, y aumentar la salud y el bienestar en los niños y los jóvenes adolescentes.

Tonucci (1997) denuncia que con frecuencia la escuela enseña todo a los estudiantes, excepto lo que les interesa a ellos mismos. Nisbet (1986) afirma que el conocimiento más importante es el de uno mismo. Es cierto que cuando se inicia el descubrimiento de uno mismo es precisamente en la etapa de Educación Inicial.

El yoga como disciplina física y mental va a contribuir en el estudiante a hacer frente al estrés, le proporcionará herramientas para resolver situaciones conflictivas y mejorará su concentración (Paz, 2012). El yoga es un conjunto de técnicas aplicadas al desarrollo integral del ser humano, métodos y actividades, mediante las cuales es posible desarrollar el estado de yoga, es decir, unión entre cuerpo, mente, espíritu y el mundo que le rodea (Guin, 2009). En este sentido, el yoga puede definirse como un conjunto de técnicas de perfeccionamiento del ser humano a nivel físico, mental y espiritual.

Bendriss (2015) defiende que el yoga es una metodología o conjunto de técnicas de desarrollo interior, cuyo objetivo es lograr la plena realización del ser humano y a través de esta metodología los niños aprenden a gestionar sus emociones, a mejorar su concentración, a liberarse de conflictos y de complejos, a incrementar sus actitudes positivas, a incrementar la interacción con los demás, a estimular su creatividad y a adquirir saludables hábitos corporales.

La práctica de yoga habitualmente va a ayudar a los estudiantes a canalizar la energía y reafirmar su autoestima, a mejorar la seguridad que tienen en sí mismos, mostrándose más sociables y menos agresivos e incrementando su alegría y espontaneidad.

El objetivo de la práctica del yoga está en función de los intereses del practicante (Hayes y Chase, 2010; Lahoza, 2012; Monro, 1997; Panebianco, Sridharan, y Ramaratnam, 2015; Subramanyam, 1989); en nuestro caso, los practicantes son estudiantes de la etapa de Educación Inicial, por lo que el objetivo será obtener la relajación, descanso y equilibrio para desarrollar su mente y aumentar su capacidad de concentración mediante el conocimiento de sí mismo y aprovechar más eficientemente su potencial.

Cada una de las posturas que adopta el cuerpo en la práctica del yoga o llamadas técnicamente asanas brindan a los estudiantes la oportunidad 
de divertirse imitando los diferentes animales que simulan diversas posturas, además, les enseñan a respirar correctamente y mediante la práctica de sus ejercicios, aprenden a relajarse. Como en cualquier otra actividad, a los estudiantes no se les debe obligar, sino que los ejercicios de yoga deben hacerse induciéndolos hacia el descanso y la distensión muscular, como una continuidad natural de las actividades, disfrutando de la relajación.

La relajación produce en el estudiante una valoración de la imagen que construye de sí mismo. Es necesario introducir desde los primeros niveles educativos ejercicios que mejoren y beneficien la situación del niño frente a su propia actividad. En la relajación, la mente se calma y el cuerpo está preparado para aprender mejor, en un estado de mayor concentración, pues logran una mayor conciencia de sus propios pensamientos y emociones (Paz, 2012). En estas condiciones, se favorece la atención, la memoria y la incorporación de nuevos conocimientos.

La práctica del yoga en el aula favorece la salud física y emocional de los nińos desde muy pequeños, que influirá en el progreso escolar e integración social. Su práctica diaria, como se ha dicho ya anteriormente, disminuye la ansiedad, aumenta la capacidad de enfrentar situaciones estresantes ayudando a mejorar la salud y a conservarla (Madhana, 2013). La eficiencia en la capacidad de aprendizaje y de reflexión incrementa la capacidad creativa, la facilidad de pensar en positivo y mejora la autoimagen.

Los estudiantes en edades tempranas tienen una increíble sensibilidad e inteligencia. Tienen una potencialidad aún intacta y una vitalidad incansable, a la vez que muestran gran curiosidad, deseo y capacidad de aprender (Morelli, 2010), por ello, la práctica del yoga es buena para que la mente del estudiante descanse e interiorice lo aprendido.

Igualmente, es sabido que los nińos de esta etapa educativa se distraen con mucha facilidad, por esta razón, es recomendable la realización de ejercicios de yoga, entre actividades, pues como consecuencia de esos ejercicios que les resultan divertidos y relajantes, consiguen relajarse y no se les hace tan aburrido la estancia en el aula. La práctica del yoga es una actividad agradable, porque no existe competencia ni con los otros, ni con uno mismo, solo existe el descubrimiento y la aceptación de sí mismo (Philizot, 1998).

Los valores del silencio y de respeto no se enseńan como una ciencia y solo se transmiten por el ambiente de contagio, educando el estilo de vida, fomentando la experiencia, por este motivo, los docentes deben estar tranquilos.

Antes de comenzar los ejercicios de yoga, hay que conseguir atraer la atención y el interés de los estudiantes, si no su aplicación no será tan efectiva.

Se deben proponer técnicas de yoga basadas especialmente en actividades lúdicas, donde a pesar de su carácter movido y juguetón, no serán ajenas a los beneficios de la tranquilidad y la serenidad. 
Es importante tener en cuenta que, a pesar de sus ventajas, no todos los estudiantes responden de la misma manera ante estos ejercicios. Los más pequeños y aquellos más extrovertidos son los más beneficiados. Es recomendable que se incorporen ejercicios de yoga variados, pues serán más motivadores y favorecerá la confianza, el aprecio y el cariño entre los estudiantes.

Los ejercicios pueden ser acompañados con música, siempre y cuando esta se adecue a la temática del juego. Se debe ser innovador, proponer temas fantásticos y jugar con la imaginación de los estudiantes.

El yoga es una buena metodología para tratar los problemas del aula pues, según Faúndez (2014), un beneficio de su práctica en el aula es la mejora de la convivencia y el bienestar. Su práctica produce un clima de armonía y buena disposición de parte de los estudiantes. Según Serra (2014), el yoga fomenta las relaciones de respeto entre los estudiantes, la tolerancia y la paciencia. Asimismo, ayuda a expresar sus emociones y sentimientos reduciendo de este modo los comportamientos agresivos.

Para dar lugar a un buen aprendizaje, la secuencia programada debe ser una labor estimulante, llevando a cabo una metodología activa con dinámicas de trabajo motivadoras dotadas de creatividad para que el proceso sea efectivo (Maestre, 2010).

El éxito de esta estrategia está en manos del docente. Debe tener confianza en sí mismo y serenidad interior, porque solo así podrá transmitir a los estudiantes seguridad.

Figura 1. El yoga en el aula de Educación Infantil

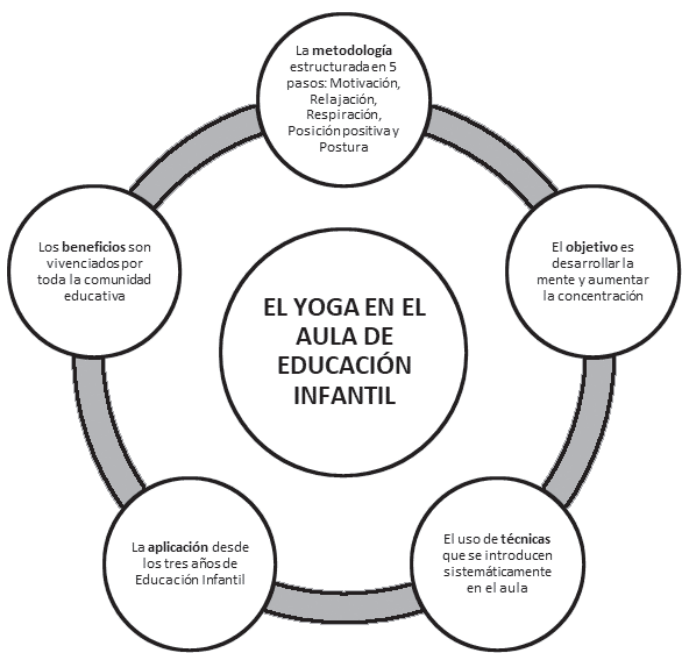

Fuente: Elaboración propia a partir de Fulán (2012) 


\subsection{Metodología del programa de yoga}

La metodología del programa de yoga en el aula seguida con los estudiantes de Educación Inicial ha sido elaborada teniendo en cuenta aspectos de la propuesta didáctica de Furlán (2012). Está repartida en sesiones de no más de media hora, donde el docente, a través de ciertas técnicas de relajación y respiración, enseña ejercicios y posturas. Estas sesiones consisten en un trabajo corporal y mental distribuido en cinco pasos.

El primer paso es la motivación. En razón de esto, es conveniente que en una clase de yoga no haya más de quince estudiantes reunidos. Estos deben sentirse cómodos y relajados. Deben vestirse con ropas holgadas, ligeras y confortables que les permitan hacer cualquier movimiento con comodidad. Las sesiones deben darse en un ambiente limpio, tranquilo, ventilado y silencioso. Se pueden utilizar colchonetas o alfombrillas apropiadas y los estudiantes pueden estar descalzos o con calcetines.

El yoga puede ser practicado a cualquier hora del día, pero el mejor horario para su práctica es por la mañana. Además, es recomendable que se evite la ingestión de alimentos sólidos dos horas antes de su práctica (Kojakovic, 2005).

El segundo paso es aprender a relajarse. Se hace concentrándose en varios sonidos durante tres minutos con luz tenue y una música suave de fondo.

El tercer paso, ya incorporados y de pie en las colchonetas, es ver una práctica hecha con estudiantes para visualizar las respiraciones. El inhalar y el exhalar por la nariz es esencial para que el estudiante pueda estirarse y obtener el equilibrio necesario para empezar a practicar las posturas del yoga.

En el cuarto paso, se trabajan las posiciones. Una vez que el grupo controle su respiración, el docente comenzará con las posturas. El objetivo de las posiciones siempre conlleva aspectos positivos, pues si se comienza desde pequeños se va tomando conciencia de su cuerpo, a conocer y poder mover cada parte de él. Se fijan más en su respiración y aprenden a permanecer en un estado de paz.

El quinto paso sería el procedimiento para la realización de algunas posturas recomendadas para nińos.

\section{Metodología}

\subsection{Objetivo de la investigación}

El objetivo de la investigación realizada es mejorar el dominio del movimiento corporal, la mejora de la memoria, la atención y la concentración, así como, la creatividad en el estudiante de Educación Inicial, utilizando la práctica del yoga en el aula al comienzo de las sesiones de clase (entrada, al centro y vuelta del recreo). 


\subsection{Metodología de la investigación}

Tomando como base el objetivo planteado en la aplicación del yoga en el aula, evaluamos individualmente la consecución del mismo de forma trimestral, es, asimismo, el proceso de enseñanza-aprendizaje, la distribución y organización espacial y el tiempo para la mejora de la intervención educativa.

Se emplearon distintas técnicas de recogida de información, tales como la observación en el aula, el registro anecdótico, el cuaderno de notas y la tabla de contenidos trimestral (instrumento creado y adaptado a las características de la investigación). La razón que justifica la variedad de instrumentos es que cada una tiene una finalidad propia y diferenciada del resto.

La complementariedad de los instrumentos empleados hace que, usados conjuntamente, la recogida de datos se produzca de un modo más fiable y válido.

Para garantizar la calidad de los datos se ha tenido en cuenta:

1. La validez, es decir, las técnicas de recogida de información han medido lo que se pretendía que midiesen.

2. La fiabilidad, pues se ha medido y recopilado la información sistemáticamente tras las sesiones de clase realizadas después de la práctica del yoga.

3. La exhaustividad, la cual significa que se han reflejado y analizado los datos con la mayor minuciosidad posible.

Para obtener la información necesaria, utilizamos los siguientes instrumentos:

- Diario de clase o anecdotario.

- Tabla de Contenidos Trimestral.

- Observación participante.

- Cuaderno de notas.

El diario de clase o anecdotario (Tabla 2), según Zabalza (2005), es el documento en el que los docentes recogen sus impresiones sobre lo que está sucediendo en sus clases o en sus aulas. El contenido del diario pude ser cualquier cosa, que en opinión de quien lo escribe, resulte destacable. En el diario de clase o anecdotario, se deja constancia de las observaciones realizadas acerca de las actuaciones más significativas del estudiante en situaciones diarias del proceso de enseńanza-aprendizaje. Preferentemente, suele recoger evidencias sobre su adaptación social y las conductas típicas relacionadas a la interacción 
de estos con el medioambiente y con el contexto social en el que se desenvuelven. En la aplicación de este instrumento, es recomendable registrar la actuación observada de forma inmediata y de manera fehaciente para no distorsionar la anécdota ocurrida. Se deben redactar los hechos observados de forma breve, clara y objetiva. Se debe también asentar de forma independiente: el incidente observado, la apreciación del docente y las sugerencias para la toma de decisiones. Es convenientemente consignar tanto los comportamientos positivos como los negativos, para tomar medidas necesarias.

Tabla 2. Anecdotario

\begin{tabular}{|c|c|c|c|c|c|}
\hline Alumno/a & \multicolumn{5}{|c|}{ Registro anecdótico/Anecdotario } \\
\hline & Criterio & \multicolumn{2}{|l|}{ Objetivo } & \multicolumn{2}{|c|}{ Técnica } \\
\hline & Comportamiento & \multicolumn{2}{|c|}{$\begin{array}{l}\text { Evaluar Comportamientos } \\
\text { en situaciones cotidianas } \\
\text { en el Centro Escolar }\end{array}$} & $\begin{array}{l}\text { Obs } \\
\text { Reg }\end{array}$ & $\begin{array}{l}\text { rvación/ } \\
\text { tro }\end{array}$ \\
\hline & \multicolumn{5}{|l|}{ Periodicidad: Diaria } \\
\hline & $\begin{array}{l}\text { Actuaciones } \\
\text { Significativas de los } \\
\text { estudiantes }\end{array}$ & $\begin{array}{l}\text { Incidente } \\
\text { observado }\end{array}$ & \multicolumn{2}{|c|}{$\begin{array}{l}\text { Apreciación de } \\
\text { docente }\end{array}$} & $\begin{array}{l}\text { Sugerencias } \\
\text { para la toma de } \\
\text { decisiones }\end{array}$ \\
\hline & \multicolumn{5}{|l|}{ Adaptación social } \\
\hline & $\begin{array}{l}\text { Conductas en la } \\
\text { interacción }\end{array}$ & & & & \\
\hline
\end{tabular}

La Tabla de Contenidos Trimestral (ver Tabla 3) es una técnica de indagación respetable y válida que, bien construida y aplicada, puede ser un instrumento muy apropiado para la recopilación de datos, permitiendo recoger información cuantificable y determinada previamente por los evaluadores. Con esta tabla se pretende conocer qué aspectos ha mejorado el estudiante tras la aplicación de las sesiones de yoga en la dinámica del aula-clase. La tabla ha sido elaborada teniendo en cuenta el área motora y de desarrollo físico, y el área social y emocional, áreas que se pueden mejorar con la práctica del yoga y que contribuyen a la mejora del área cognitiva y, del habla y la comunicación. 


\section{Tabla 3. Contenido trimestral}

Nombre/

Apellidos:

Indicadores de contenidos

Conseguido No conseguido

Ha mejorado la expresión de sentimientos, emociones, preferencias e intereses.

Presta atención y tiene mayor interés por lo que se le comunica.

Descubre las posibilidades motrices del propio cuerpo, coordinando y controlando movimientos cada vez con mayor precisión.

Identifica las necesidades básicas de su cuerpo, como la respiración y la relajación.

Identifica diferentes posturas y movimientos corporales.

Muestra una imagen ajustada y positiva de sí mismo.

Pide ayuda cuando lo necesita.

Muestra mayor autonomía, esfuerzo y satisfacción en la realización de algunas actividades.

Canaliza su energía y reafirma su autoestima.

Se siente más seguro de sí mismo, expresándose con mayor naturalidad y disfrutando con las actividades que se le proponen.

Es más sociable, menos agresivo y se relaciona con los demás, ajustando su conducta a distintas situaciones.

Es más alegre y espontáneo.

Está más relajado, equilibrado y descansado.

Ha mejorado en el aumento de la capacidad de concentración, escuchando con interés y asimilando ideas básicas.

Tiene mejor autoconocimiento y muestra interés por reconocer las curiosidades de su cuerpo.

Ha aumentado su capacidad creativa.

Ha disminuido la ansiedad.

Tiene un pensamiento más positivo. 
Diferentes autores consideran en la investigación la práctica educativa. La observación participante se presenta como una herramienta para la recogida de información (Greenwood, 2000; Hilgers, 2013; Kawulich, 2005) La observación participante es una herramienta útil para recabar información, ya que proporciona al docente métodos para revisar expresiones no verbales de sentimientos, determinan quién interactúa con quién, permiten comprender cómo los estudiantes se comunican entre ellos, y verifican cuánto tiempo se está gastando en determinadas actividades (Schmuck y Schmuck, 1990, Schmuck, 1997)

Siguiendo a Pérez (1998) en nuestro caso, la observación tiene carácter participante por cuanto es el propio docente que investiga quien observa y participa de la vida del grupo, conversando con sus miembros y estableciendo relaciones con ellos.

Según Goetz y LeCompte (1988), la observación participante es considerada como la principal técnica etnográfica de recogida de datos, en la cual el investigador pasa todo el tiempo posible con los individuos que estudia, viviendo como ellos.

El proceso de investigación a través de la observación participante nos ha brindado la posibilidad de incorporar todo aquello que pudiera resultar interesante para nuestra investigación, aun cuando no lo habíamos incluido previamente en el momento de la planificación.

Las observaciones han sido registradas en un cuaderno de notas, el cual nos proporciona un registro formal, por escrito, de nuestro propio trabajo y pensamiento. El cuaderno de notas se configura como un instrumento óptimo para la observación de fenómenos desde dentro y en relación con los contextos en los que se producen. El carácter abierto del cuaderno de notas ofrece la posibilidad de incluir lo inesperado, lo que previamente no estaba programado, constituyendo así un marco flexible para la recogida de datos (McKernan, 2001). En este hemos recogido las situaciones e informaciones más destacadas de cada sesión.

Los instrumentos utilizados para la recogida de información son unas herramientas que ayudan a los docentes que imparten docencia en las aulas de Educación Inicial en las que se aplica el yoga, a pensar críticamente sobre los problemas a los que se enfrentan, sobre las prácticas diarias de su actuación y sobre las ventajas e inconvenientes de la introducción del yoga. Son herramientas para pensar con claridad, pues nos permiten examinar sistemáticamente lo que acontece en el aula-clase, establecer estándares con las mejores prácticas y reflejar el progreso hacia los objetivos que son mensurables. Los datos recogidos nos ayudan a la comprensión y nos facilitan la toma de decisiones. 
En referencia a la muestra elegida para llevar a cabo la investigación, es no probabilística y se conforma con el grupo de trabajo que estaba formado por la totalidad de los estudiantes matriculados (72 estudiantes) en la etapa de educación inicial, en edades comprendidas entre 3 y 6 ańos, de un centro educativo público de inicial y primaria de una zona rural. La investigación se realiza durante un curso académico.

Tabla 1. Muestra participante

\begin{tabular}{cccc}
\hline Edad & Masculino & Femenino & Total \\
\hline 3 & 16 & 7 & 23 \\
4 & 17 & 8 & 25 \\
5 & 17 & 7 & 24 \\
\hline \multicolumn{2}{r}{ Total } & 72 \\
\hline
\end{tabular}

La muestra estuvo constituida por grupos heterogéneos en los que predominó la inquietud y la actividad; esto se observa en la utilización de un tono de voz muy elevado, a veces una escasa concentración y poca conciencia de grupo, lo que afecta al trabajo de rutinas influyendo así en el rendimiento académico, en su integración y socialización. El trabajo de rutinas es crucial en la Educación Inicial, pues les ayuda a identificar la acción que tienen que realizar, para que así puedan interiorizarla y sentirse más seguros.

El origen de los estudiantes es muy variado con unas características familiares y una procedencia (la mayoría vienen de guardería) muy distinta. Así, el periodo de acogida y la práctica del yoga en estas edades son fundamentales no solo para lograr un ambiente cálido y acogedor, sino para favorecer estas diferencias, convertirlos en grupos más relajados, con mejora de hábitos de trabajo y con autonomía personal.

\subsection{Rutina de las sesiones}

Hay muchas maneras de practicar el yoga tanto como corrientes y estilos. En nuestro proyecto de yoga, coincidimos en que todas parten del mismo principio: bienestar y equilibrio. Para elaborar la rutina de sesiones, se ha tenido en cuenta libros como El yoga para niños de Kojakovic (2005). Con el ejemplo de rutina de sesiones que se describe a continuación se puede comprobar cómo el estudiante aprende las secuencias de relajación y respiración.

La manera más divertida que encontramos para introducir el yoga en estudiantes de Educación Inicial es la de imitar a diferentes animales que simulaban las diversas posturas, eso sí, teniendo especial cuidado en no exigirles 
que repitieran una postura una y otra vez, puesto que podría convertirse en algo muy aburrido para ellos.

Estas posturas enseñaron a respirar correctamente a los estudiantes de Educación Inicial que comprobaron que el inhalar y el exhalar por la nariz era necesario para poder estirarse mejor e incluso obtener mejor equilibrio en las distintas posiciones.

Veremos una práctica similar al trabajo mental y corporal que realizamos con estos estudiantes durante todo un curso escolar, dos o tres días en semana.

- Primero: Tumbados

En alfombrillas o colchonetas, vamos a relajarnos dos o tres minutos. Nos concentraremos en varios sonidos, con luz tenue y una música suave de fondo.

- Segundo: Sentados con piernas estiradas a lo largo del cuerpo Hacemos ejercicios de calentamiento, sobre todo: pies, piernas, rodilla, cadera, hombros, cuello y cabeza.

- Tercero: Respiraciones (en cada postura inhalaremos o exhalaremos) Postura de oración (exhalar), postura de brazos levantados (inhalar), postura de manos y pies (inhalar), postura de paso extendido (exhalar), postura de la montańa (retener), saludo al sol (exhalar), postura de la cobra (inhalar), paso extendido (exhalar), postura de manos y pies (inhalar), brazos estirados (exhalar). Y volver poco a poco a la postura de pie. Bajar los brazos al lado del cuerpo y juntar las palmas de las manos.

- Cuarto: Posiciones de yoga

Recordar con estas posiciones de yoga aprendidas por los estudiantes de Educación Inicial imitaban a animales y que se incorporaron de manera progresiva de dos en dos, y siguiendo siempre el mismo orden. Cuando los estudiantes aprendían las dos primeras, pasábamos a las siguientes. Posiciones y posturas realizadas: postura de estrella de mar, de serpiente, de foca, de búho, de flor, de pájaro, de columpio, de gato, de tortuga, de ardilla, de grillo, de hoja, de mecedora y de hamaca.

- Quinto: Relajación

Finalmente, hacen unas respiraciones profundas para relajarse y estar tranquilos para las siguientes actividades escolares.

Los recursos utilizados, en la rutina de sesiones, están dirigidos a crear una atmosfera de paz, es decir: 
- Un aula sin distractores (sin mucha decoración, con poca luz, ventilada, aislada de ruidos, etc.) y con materiales que ayuden al estudiante de Inicial a sentirse cómodo: colchonetas, cojines, mantas. Deben vestirse holgados, ligeros, confortables que les permita hacer cualquier movimiento con comodidad.

- Un equipo de sonido

- Un ordenador

- Música: sonidos ambientales de la naturaleza, cantos tibetanos, música clásica.

- Cuentos: cuentos que desarrollen su potencial creativo y su capacidad de resolver problemas y superar retos.

- Videos del Portal Educativo de Castilla-La Mancha: Materiales y tareas de varios autores.

\section{Discusión de Resultados}

Para el análisis de los resultados, se ha llevado a cabo un grupo de discusión con todo el equipo docente que imparte clase en la etapa de Educación Inicial y se ha analizado toda la información reflejada en los instrumentos de recogida de datos. Se han tenido como referencia los indicadores de la Tabla de contenidos Trimestral (Tabla 3).

Se observó que tras una evaluación inicial en los estudiantes que forman parte de la muestra:

El $34 \%$ de los estudiantes:

- Presta atención y tienen interés por lo que se les comunica.

- Muestran una imagen ajustada y positiva de sí mismo.

- Canalizan su energía y reafirman su autoestima.

- Se sienten seguros de sí mismos.

- Son alegres y espontáneos.

El $38 \%$ de los estudiantes:

- Muestran una buena expresión de sentimientos, emociones, preferencias e intereses.

- Tienen una adecuada capacidad creativa.

- Tienen la capacidad de concentración ante las tareas que se realizan.

El $42 \%$ de los estudiantes:

- Pide ayuda cuando lo necesita.

- Muestra nerviosismo y/o ansiedad. 
Mientras que en las evaluaciones aplicadas durante el proceso y al finalizarlo, se ha encontrado que el $100 \%$ de los estudiantes

- Han ido descubriendo las posibilidades motrices de su propio cuerpo, coordinando y controlando los movimientos con mayor precisión.

- Han sido capaces de identificar diferentes posturas y movimientos corporales.

- Han mostrado mayor autonomía, esfuerzo y satisfacción en la realización de algunas actividades.

- Son más sociables, menos agresivos y se relacionan ajustando sus conductas a distintas situaciones.

- Están más relajados, equilibrados y descansados.

- Tienen un mejor autoconocimiento y muestran interés por reconocer las curiosidades de su cuerpo.

- Tienen un pensamiento más positivo.

Solo el 28\% de los estudiantes es capaz de identificar las necesidades básicas de su cuerpo como son la respiración y la relajación.

Figura 2. Identifica las necesidades básicas de su cuerpo, como la respiración y la relajación

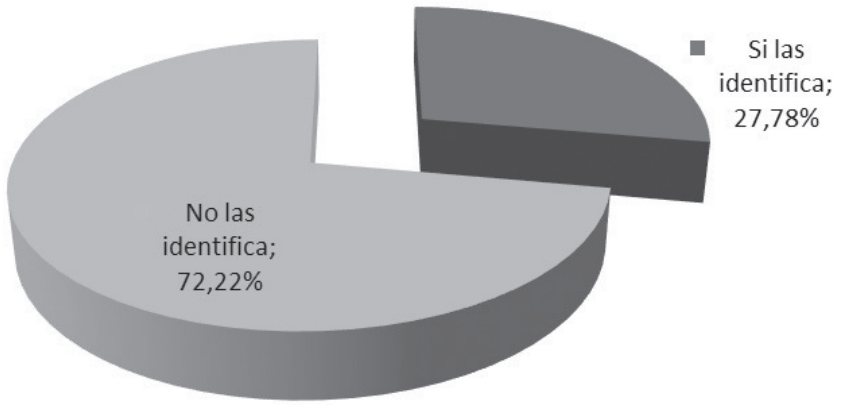

El 98\% de los estudiantes:

- Prestan más atención y tienen mayor interés por lo que se les comunica.

- Muestran una imagen más ajustada y positiva de sí mismo.

- Canalizan mejor su energía y reafirman su autoestima.

- Se expresan con mayor naturalidad y disfrutan con las actividades que se les proponen.

- Son más alegres y espontáneos.

- Han aumentado la capacidad de concentración, escuchan con más interés y asimilan mejor las ideas básicas. 
El 94\% de los estudiantes ha mejorado:

- La expresión de sentimientos, emociones, preferencias e intereses.

- Su capacidad creativa.

El 87\% ahora:

- Pide ayuda cuando lo necesita.

- Tiene menos ansiedad.

Los resultados han sido altamente positivos, lo que nos ha permitido comprobar los múltiples beneficios que el yoga aportó tanto en el desarrollo físico, cognitivo, social y emocional. Se ha transmitido optimismo a los estudiantes de manera que los estudiantes mostraban más seguridad y tranquilidad en el aula.

También se evaluó la práctica educativa del docente comprobándose que tanto el proceso seguido como la actuación, así como, su nivel de idoneidad, el conocimiento del sujeto y su relación con el espacio fueron satisfactorios. Se han aprovechado los medios con los que se contaba, se ha sabido motivar y animar a los estudiantes y conseguir que aprendan.

\subsection{Dificultades de la aplicación del programa}

La aplicación de nuestro proyecto de yoga encontró una primera dificultad que fue el lugar. El aula tenía demasiados estímulos visuales, lo que facilitaba la distracción de los estudiantes de Educación Inicial, por lo que se tuvo que redecorar y minimizar esos estímulos.

Otra dificultad es que suele haber un solo docente para la práctica del yoga, siendo complicado estar dirigiendo a los estudiantes cuando ellos necesitan observar e imitar al docente que debe hacer los ejercicios al mismo tiempo como ejemplo.

El horario es crucial. Es conveniente aplicarlo a primera hora de la mañana en el aula, como una rutina, pero también entre actividades para que los estudiantes adopten actitudes más tranquilas y de autocontrol en el resto de la jornada.

En la primera sesión de yoga, los estudiantes más inquietos solo lograban estar concentrados treinta segundos, pero con el tiempo se consiguió que lo estuvieran hasta media hora.

Podemos encontrar como inconveniente que los estudiantes de Educación Inicial puedan carecer de agilidad y estar demasiado rígidos, pero se verá que es algo fácilmente superable mediante la práctica regular del yoga. 
En ocasiones, la dificultad puede venir por la actitud competitiva de algunos estudiantes hasta que perciben que solo es un juego, donde incluso el estudiante más tímido pierde su miedo ante los demás o, en el caso contrario, el más inquieto o activo se relaja.

Es muy importante también que los docentes se encuentren relajados en el momento de la sesión, puesto que les podemos transmitir el nerviosismo por el tono de voz.

\section{CONCLUSIONES Y RECOMENDACIONES}

La práctica del yoga en las escuelas es una actividad que debería formar parte de la educación integral y ser integrada en el plan de estudios escolar explícito de las áreas a enseñar, y de las actividades y hábitos de vida saludables que tratan de promover; previene y reduce el estrés, ansiedad, el miedo, la inseguridad, la desconfianza (Marian Garfinkel y Schumacher, 2000)

La práctica del yoga, mediante actividades lúdicas, ayuda al estudiante a prepararse para convivir y participar activamente en la vida social del aula, la educación para la salud, la creación de hábitos creativos y sanos (Subramanyam, 1989).

El yoga debe practicarse desde los primeros niveles (Lahoza, 2012; White, 2009), porque es precisamente en la edad en que resulta más educable, pero hay que tener en cuenta que las prácticas o actividades aisladas son ineficaces. Es necesario un plan global elaborado desde un análisis de la realidad concreta, es decir, un plan a partir del conocimiento de las características de los estudiantes, del contexto escolar y del entorno más cercano.

La práctica del yoga no debe ser ajena a las necesidades y problemas específicos de los estudiantes y de los grupos ni de su edad. La planificación de cada una de las sesiones debe tener explícitas las metas u objetivos, como soluciones posibles a los problemas o inquietudes detectadas y, debe señalar con precisión y claridad el conjunto de actividades que han de ser ejecutadas para alcanzar cada uno de nuestros objetivos o metas.

Durante la práctica de yoga en Educación Inicial, no se debe forzar a los estudiantes a hacer los ejercicios, pues el enfoque principal es que se diviertan y que tengan libertad de hacer lo que puedan.

Tras la puesta en práctica de cada una de las sesiones de yoga en el aula debemos evaluar el proceso de enseñanza. Esta evaluación se puede llevar a cabo teniendo en cuenta una serie de preguntas generales como las siguientes: ¿La motivación fue satisfactoria? ¿Contaba con los recursos necesarios? ¿Los estudiantes respondieron satisfactoriamente? ¿La planificación del tiempo fue correcta? ¿Se han tenido en cuenta los ritmos generales y particulares? 
¿El espacio físico permitió la actividad correctamente? ¿Los materiales han sido adecuados, motivadores, seguros? Durante la sesión práctica se podrían tener en cuenta otro tipo de preguntas como: ¿Se mantuvo la motivación del alumnado? ¿La participación del alumnado ha sido normal? ¿Las actividades han conseguido lograr los objetivos? ¿Se han incorporado modificaciones sobre lo planificado? ¿La relación entre los alumnos ha sido satisfactoria? ¿Hubo complejidad en algún tipo de actividad? ¿Surgió alguna problemática que afectase a todo el grupo? Después de la sesión podríamos reflexionar sobre: ¿La satisfacción en la realización fue gratificante? ¿Qué les gusto hacer? ¿Qué es lo que menos les gustó? ¿Qué fue lo que les costó más? ¿Qué podríamos haber hecho? ¿Qué deberíamos evitar?

En cuanto a la evaluación del proceso aprendizaje o contribución de la práctica del yoga al desarrollo del estudiante, se puede tener en cuenta a modo de ejemplo la Tabla de Contenidos Trimestral (Tabla 2).

Es importante que la práctica del yoga sea impartida por maestros especializados en estas actividades, pues podría resultar contraproducente si no se tienen los conocimientos y la experiencia adecuada.

Se puede considerar que el yoga es una excelente actividad integradora de los aspectos emocional, cognitivo y creativo. Es una buena opción para lograr la unión de juego y desarrollo físico y mental en edades tempranas.

\section{REFERENCIAS BIBLIOGRÁFICAS}

Bendriss, Y. (2015). Yoga en familia. Guía práctica para padres y educadores. España: Arcopress.

Boman, P. y Yates, G. C. (2001). Optimism, hostility, and adjustment in the first year of high school. British Journal of Educational Psychology, 71(3), 401-411. https://doi.org/10.1348/000709901158587

Chao, C. Y., Hwu, S. L. y Chang, C. C. (2011). Supporting interaction among participants of online learning. Turkish Online Journal of Educational Technology, 10(4), 311-319.

Dawson, M. y Pooley, J. A. (2013). Resilience : The role of optimism, perceived parental autonomy support and perceived social support in first year university students. International of Education and Training Studies, 1(2), 38-49. https://doi.org/10.11114/jets.v1i2.137

Division for Early Childhood (2014). DEC recommended practices in early intervention/early childhood special education 2014. Developed by the DEC Recommended Practices Commission.

Division for Early Childhood (2016). DEC Recommended Practices with Examples Anexo de DEC Recommended Practices (April 2014) which 
includes accompanying examples for each practice to further explain the successful implementation of the practice. Developed by the DEC Recommended Practices Commission.

Faúndez, J. (2014). Estrategias no tradicionales en la educación diferencial y en procesos de mediación personalizada. Paulo Freire. Revista de Pedagogía Crítica, 13(15), julio.

Furlán, E. (2012). Yoga para niños y niñas. Barcelona: Editorial Hispano Europea.

Garfinkel, M. y Schumacher, H. R. (2000). Yoga. Rheumatic Disease Clinics of North America, 26(1), 125-132. https://doi.org/10.1016/ S0889-857X(05)70126-5

Gillham, J. E., Shatté, A. J., Reivich, K. J. y Seligman, M. E. P. (2001). Optimism, pessimism, and explanatory style. En E.D. Chang (ed.), Optimism pessimism implications for theory research and practice (pp. 53-75). https://doi. org/10.1037/10385-003

Gillham, J. y Reivich, K. (2004). Cultivating Optimism in Childhood and Adolescence. The Annals of the American Academy of Political and Social Science, 591(1), 146-163. https://doi.org/10.1177/0002716203260095

Goetz, J. P. y LeCompte, M. D. (1988). Etnografía y diseño cualitativo en investigación educativa. Madrid: Morata.

Greenwood, D. (2000). De la observación a la investigación-acción partipativa: una visión crítica de las prácticas antropológicas 1. Revista de Antropología Social, (9), 27-49.

Guin, J. (2009). Karma Yoga, una vida saludable. Londres: Editorial Blume.

Hayes, M. y Chase, S. (2010). Prescribing Yoga. Primary Care: Clinics in Office Practice, 37(1), 31-47. https://doi.org/10.1016/j.pop.2009.09.009

Hernangómez, L. (2002) Variables de influencia temprana en la génesis del optimismo. Revista de Psicopatología y Psicología Clínica, 7(3), 227-242. https://doi.org/10.5944/rppc.vol.7.num.3.2002.3936

Hilgers, M. (2013). Observation participante et comparaison. Anthropologie et Sociétés, 37(1), 97. https://doi.org/10.7202/1016149ar

Hoy, A. W., Hoy, W. K. y Kurz, N. M. (2008). Teacher's academic optimism: The development and test of a new construct. Teaching and Teacher Education, 24(4), 821-835. https://doi.org/10.1016/j.tate.2007.08.004

Janzen, H. L., Hildebrand, D. K., Kaufmann, L. y Saklofske, D. H. (2001). Depresión en los niños. National Association of School Psychologist, 187-190.

Kawulich, B. B. (2005). La observación participante como método de recolección de datos. Forum Qualitative Social Research, 6(2), Art. 43.

Kojakovic, M. (2005). El yoga para niños. Santiago de Chile: Random House Mandadori, S. A. 
Lahoza, L. I. (2012). Yoga para nińos: beneficios, metodología y aplicación práctica [revista digital]. Revista Artista Digital, (25), 10-19. Retrieved from http:// www.afapna.es/web/aristadigital/archivos_revista/2012_octubre_2.pdf

Maestre, J. (2010). Yoga para niños. Málaga: Aljibe.

Marujo, H. Á., Neto, L. M. y Perlorio, M. F. (2003). Pedagogía del optimismo. Guía para lograr ambientes positivos y estimulantes. Madrid: Narcea.

Marujo, H. Á. y Neto, L. M. (2010). Psicologia Comunitária Positiva: Um exemplo de integraçáo paradigmática com populações de pobreza. Analise Psicologica, 28(3), 517-525. https://doi.org/10.14417/ap.355

Marujo, H. Á., Neto, L. M., Caetano, A. y Rivero, C. (2007). Revolução positiva: Psicologia positiva e práticas apreciativas em contextos organizacionais. Comportamento Organizacional e Gestão, 13(1), 115-136. Recuperado de http://www.scielo.gpeari.mctes.pt/pdf/cog/v13n1/v13n1a07.pdf

McKernan, J. (2001). Investigación-acción y curriculum. Madrid: Morata.

Monro, R. (1997). Yoga therapy. Journal of Bodywork and Movement Therapies, 1(4), 215-218. https://doi.org/10.1016/S1360-8592(97)80047-2

Morelli, M. (2010). El yoga en la infancia. Ejercicios para divertirse y crecer con salud $y$ armonía. Madrid: Narcea

National Association for the Education of Young Children - NAEYC (2009). Práctica Apropiada para el Desarrollo en Programas para la Primera Infancia para la Atención de Niños desde el Nacimiento hasta los 8 Años de Edad. Declaración de Posición de NAEYC. Práctica Apropiada para el Desarrollo, 3ra edición. Recuperado de https://www.naeyc.org/files/naeyc/ file/positions/Spanish\%20DAP\%20position\%20statement\%20(1).pdf

Nisbet, J. y Schucksmith, J. (1986). Estrategias de aprendizaje. Madrid: Santillana. Panebianco, M., Sridharan, K. y Ramaratnam, S. (2015). Yoga for epilepsy. The Cochrane Database of Systematic Reviews. https://doi.org/ 10.1002/14651858.CD001524.pub2

Paz, R. (2012). Yoga para niños, técnicas, asanas y rutinas. Perú: Ediciones Mirbet.

Pérez, M. G. (1998). Investigación cualitativa. Retos e interrogantes. II Técnicas y análisis de datos. Madrid: La Muralla.

Philizot, H. (1998). Despertar la atención - 30 sesiones de relajación y yoga en la escuela inicial. Barcelona: Editorial Paidotribo.

Ruthig, J. C., Haynes, T. L., Perry, R. P. y Chipperfield, J. G. (2007). Academic optimistic bias: Implications for college student performance and wellbeing. Social Psychology of Education, 10(1), 115-137. https://doi. org/10.1007/s11218-006-9002-y 
Schmuck, P. y Schmuck, R. (1990). Democratic Participation in SmallTown Schools. Educational Researcher, 19(8), 14-19. https://doi. org/10.3102/0013189X019008014

Schmuck, R. (1997). Practical action research for change. Arlington Heights, IL: IRI/Skylight. Training and Publishing.

Seligman, M. E. P., Ernst, R. M., Gillham, J., Reivich, K. y Linkins, M. (2009). Positive education: positive psychology and classroom interventions. Oxford Review of Education, 35(3), 293-311. https://doi.org/ $10.1080 / 03054980902934563$

Serra, L. (2014). El yoga educa. Recuperado de http://www.elyogaeduca.com/ lidia-serra-lopez/?lang=es

Subramanyam, S. (1989). Yoga for health. Yoga Mimamsa, 28, 60-64.

Telles, S., Gaur, V. y Balkrishna, A. (2009). Effect of a Yoga Practice Session and a Yoga Theory Session on State Anxiety. Perceptual and Motor Skills, 109(3), 924-930. https://doi.org/10.2466/pms.109.3.924-930

Tonucci, F. (1997). La ciudad de los niños. Madrid: Fundación Germán Sánchez Ruiperez.

White, L. S. (2009). Yoga for Children. Pediatric Nursing, 35(5), 296-297. https:// doi.org/10.5667/tang.2011.0008

Whitebread, D. y Basilio, M. (2012). Emergencia y desarrollo temprano de la autorregulación en niños preescolares. Profesorado. Revista de Currículum y Formación del Profesorado, 16(1), 15-34.

Zabalza, M.A. (2005). Diarios de clase. Un instrumento de investigación y desarrollo profesional. Madrid: Narcea. 